Check for updates

Cite this: RSC Adv., 2021, 11, 27782

\title{
Visible-light synthesis of 4-substituted-chroman- 2-ones and 2-substituted-chroman-4-ones via doubly decarboxylative Giese reaction $\uparrow$
}

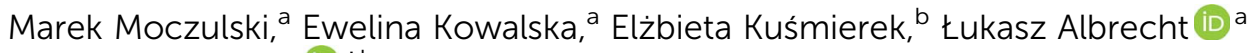 \\ and Anna Albrecht (DD *b
}

Doubly decarboxylative, photoredox synthesis of 4-substituted-chroman-2-ones and 2-substitutedchroman-4-ones is described. The reaction involves two independent decarboxylation processes: the first one initiating the cycle and the second completing the process. Visible light, photoredox catalyst, base, anhydrous solvent and inert atmosphere constitute the key parameters for the success of the developed transformation. The protocol proved applicable for coumarin-3-carboxylic acids and chromone-3-carboxylic acids as well as $N$-(acyloxy)phthalimide which served as precursors of the corresponding alkyl radicals.

Received 4th August 2021

Accepted 6th August 2021

DOI: 10.1039/d1ra05914a

rsc.li/rsc-advances

the potential of this method has been confirmed in the Giese reaction with various electron-poor olefins. ${ }^{10}$

Decarboxylative Michael reaction that involves the addition to carboxylic-acid-activated olefins followed by decarboxylation reaction constitutes a powerful synthetic tool. ${ }^{11}$ Coumarin-3carboxylic acids 2 and chromone-3-carboxylic acids 4 are useful reactants participating in this reaction opening access to biologically relevant chroman-2-ones 3 and chroman-4-ones 5. ${ }^{12,13}$ Recently, doubly decarboxylative reactions involving these reagents have also been developed. ${ }^{14}$ Surprisingly, decarboxylative Giese reaction with carboxylic-acid-activated olefins has not been a subject of studies so far. Herein, we report the first photocatalytic, doubly decarboxylative Giese reaction ata such as 4'-hydroxy-5,6,7-trimethoxyflavanone are reported to have antimycobacterial activity. ${ }^{6}$

The addition of free radicals to electron-deficient olefins is known in the literature as Giese reaction (Scheme 2). ${ }^{7}$ Recently, owing to the development of photocatalysis, the synthetic potential of this and related reactions has been vastly expanded. ${ }^{8}$ Advancements in this field arises from the development of photo-initiated methods allowing for free radical formation under mild and non-toxic conditions. An interesting way to generate free radicals involves the usage of $N$-(acyloxy) phthalimides. ${ }^{9}$ The formation of free radical is initiated by oneelectron reduction with subsequent decarboxylation. Recently,

'Institute of Organic Chemistry, Faculty of Chemistry, Lodz University of Technology, Żeromskiego 116, 90-924 Łódź, Poland

${ }^{b}$ Institute of General and Ecological Chemistry, Faculty of Chemistry, Lodz University of Technology, Żeromskiego 116, 90-924 Eódź, Poland. E-mail: anna.albrecht@p.lodz. $p l$

$\dagger$ Electronic supplementary information (ESI) available: See DOI: 10.1039/d1ra05914a

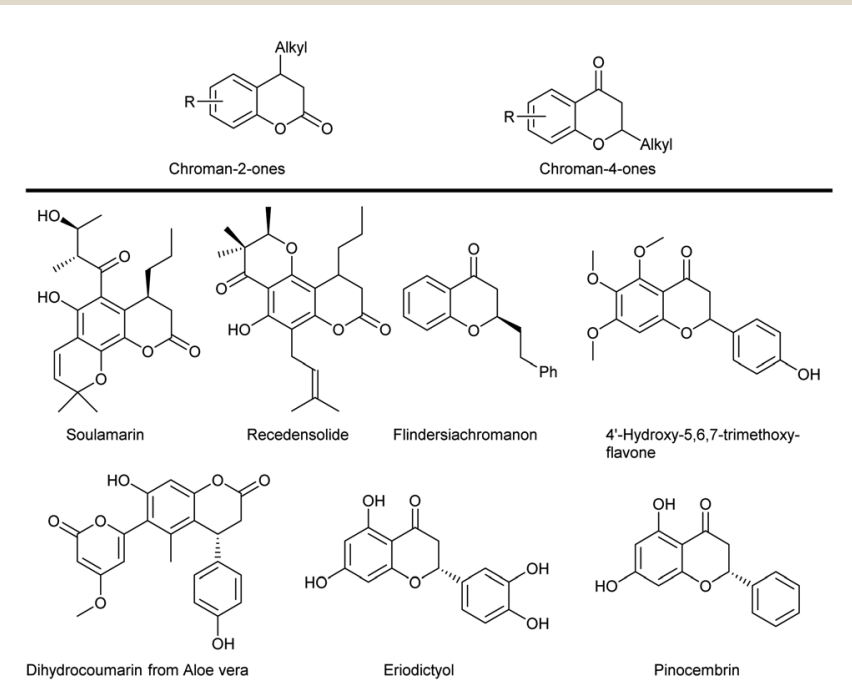

Scheme 1 The importance of chroman-2-ones and chroman-4ones. 


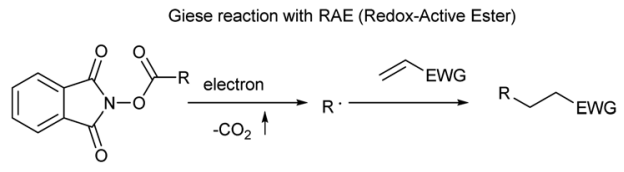

Decarboxylative Michael reaction

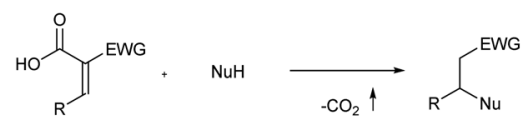

Synthetic objectives of our approaches

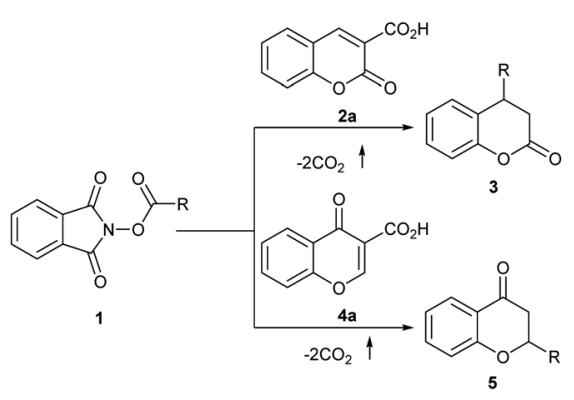

Scheme 2 The importance of decarboxylative approaches in organic synthesis and the synthetic objectives of our study.

which is applicable to electron poor carboxylic acids. The developed strategy benefits from mild reaction conditions.

Optimization studies were performed using coumarin-3carboxylic acid 2a and 1,3-dioxoisoindolin-2-yl cyclohexane carboxylate 1a (NHPI esters) as model substrates (Table 1). Initial experiments were performed in $\mathrm{CH}_{2} \mathrm{Cl}_{2}$ in the presence of DIPEA as a donor of electron and base the corresponding photoredox catalyst under LED irradiation (with the light source of suitable wavelength) under inert atmosphere. In the first part of the optimization studies, the catalytic activity of eight different photoredox catalysts was tested (Table 1, entries 1-8). When 9-mesityl-10-methylacridinium tetrafluoroborate $\mathbf{6 a}$, chloranil $\mathbf{6 b}$ or $6 \mathbf{h}$ were used, the formation of target product 3aa was not observed (Table 1, entries 1 and 2). The best yield was obtained in the presence of $\mathrm{Ru}\left(\mathrm{bpy}_{3}\right)_{2}\left(\mathrm{PF}_{6}\right)_{2}$ 6f (Table 1, entry 6). In the course of further studies, the effect of the solvent on the reaction outcome was evaluated (Table 1, entries 9-13). The use of $\mathrm{CHCl}_{3}$ did not ensure the product formation (Table 1, entry 9). Similar effect was observed in THF and toluene (Table 1, entries 10 and 11). The desired reaction took place also in acetonitrile and DMF, however, lower yield than in the case of $\mathrm{CH}_{2} \mathrm{Cl}_{2}$ was obtained (Table 1, entries 12 and 13). Subsequently, the effect of base on the reaction outcome was evaluated (Table 1, entries 14 and 15). When triethylamine was used, the yield of the reaction decreased (Table 1, entry 14) and the application of quinine did not result in the product 3aa formation (Table 1, entry 15). In the course of further studies control experiments were performed (Table 1, entries 16-20). The reaction did not proceed in the absence of neither photoredox catalysts (Table 1, entry 16, presumably electron donor-acceptor complex of DIPEA with NHPI esters was not efficiently formed in this case), nor a base (Table 1, entry 17). ${ }^{9 a}$ Similar effect was observed
Table 1 Doubly decarboxylative Giese reaction - optimization studies involving coumarin-3-carboxylic acid $2 \mathrm{aa}^{a}$

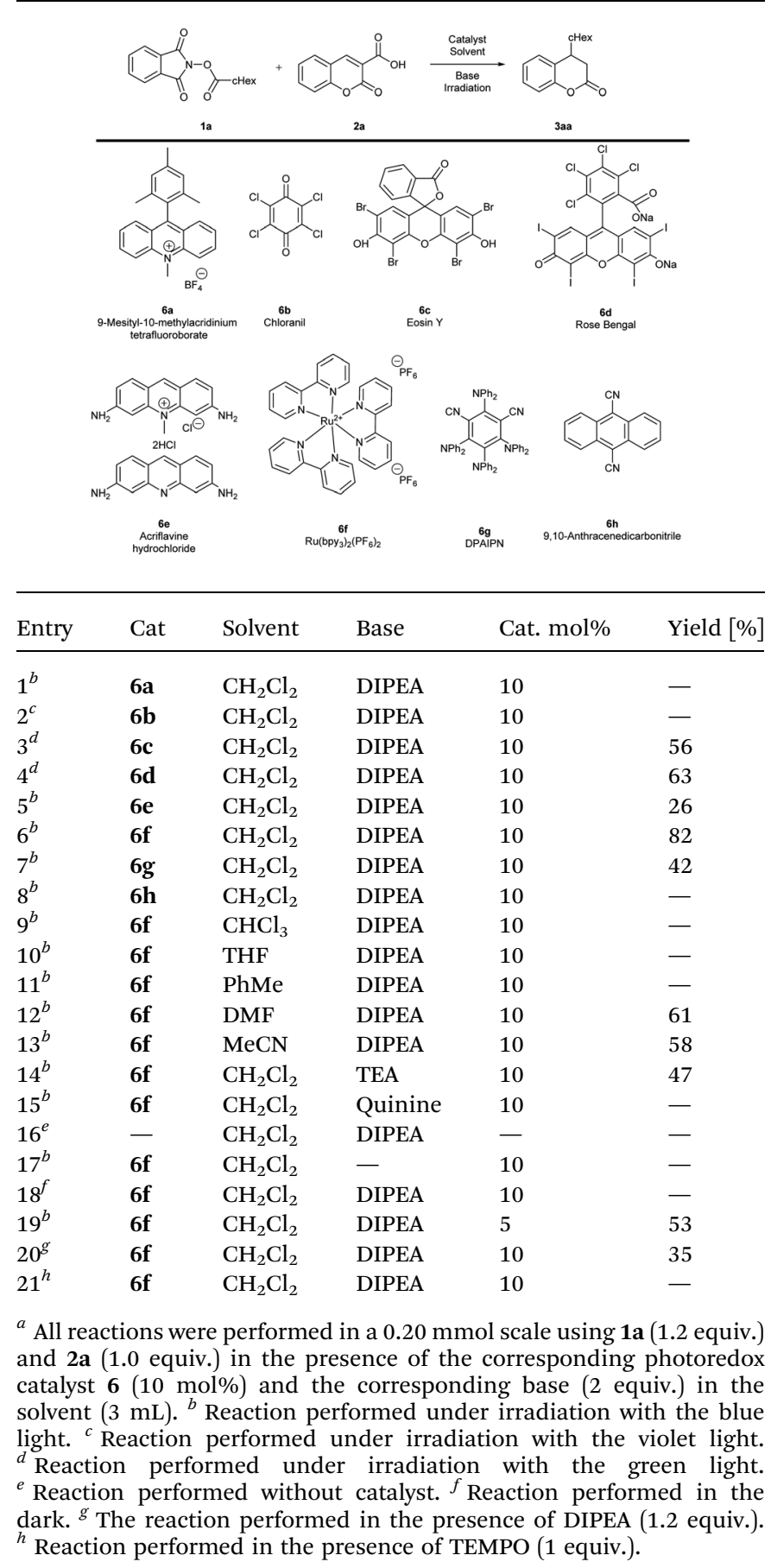

when the transformation was attempted in the dark (Table 1, entry 18), thus confirming the crucial effect of photocatalyst and the source of light on the reaction outcome. The amount of the photocatalyst $6 \mathbf{f}$ was possible to be lowered to $5 \mathrm{~mol} \%$ resulting in decrease of yield to $53 \%$ after 24 hours (Table 1, entry 19). On the other hand, it was possible to perform the reaction with DIPEA (1.2 equiv.) but the yield of the reaction decreased (Table 1 , entry 20). Noteworthily, the reactivity was quenched in the 
presence of TEMPO confirming the radical mechanism of the process (Table 1 , entry 21 ).

With the optimized reaction conditions in hand (Table 1, entry 6), the applicability of the developed methodology was studied (Schemes 3 and 4). Initially, various coumarin-3carboxylic acids 2a-i were tested (Scheme 3). Acids 2bd bearing electron-donating groups on the aromatic ring provided products 3ab-ad with higher yields. Similarly, carboxylic acids 2e-i with electron-withdrawing groups also provided products with good yields. The lowest yield was obtained for coumarin-3-carboxylic acid $\mathbf{2 h}$ with chlorine substituent in the 8-position. In the second stage of the scope studies various $N$-(acyloxy)phthalimides 1a-e were investigated (Scheme 3). $N$-(Acyloxy)phthalimides 1a-e that served as precursors of secondary and tertiary alkyl radicals turned out to be suitable components in the developed reaction. Target products 3ba-ea were efficiently formed. However, the use of primary radicals turned out to be problematic. In the case of benzylic radical precursor, a dimerization of the corresponding radical was faster than the addition to the electrophile $\mathbf{2 a}$.

In the second part of our studies, chromone-3-carboxylic acids 4 were tested as acceptors in the doubly decarboxylative Giese reaction employing the same conditions that were used in the case of coumarine-3-carboxylic acids 2 (Scheme 4 ). The use of $\mathbf{4 b}-\mathbf{d}$ bearing electron-donating groups provided $\mathbf{5 a b}, \mathbf{5 a c}$, 5ad with good results. On the other hand, the reaction of chromone-3-carboxylic acids $\mathbf{4 e - i}$ with electron-withdrawing groups provided products 5ae-5ai with slightly lower efficiency. Chromone-3-carboxylic acid $\mathbf{4 h}$ bearing two substituents with opposite electronic effects was also well-tolerated. Other alkyl radical precursors $\mathbf{1 b}$-e were also utilized in the reaction with chromone-3-carboxylic acid 4a. The tert-butyl substituted
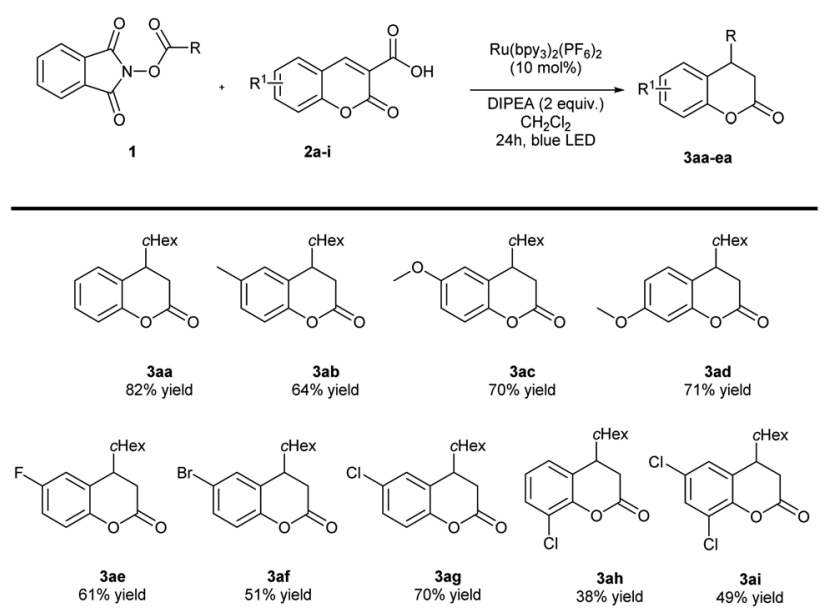

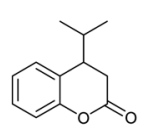

$3 \mathrm{ba}$
$45 \%$ yield

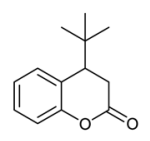

$3 \mathrm{ca}$
$60 \%$ yield

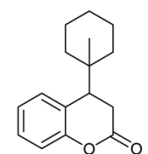

$3 \mathrm{da}$
$58 \%$ yield

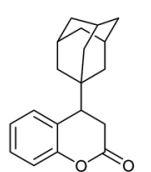

3 ea
$68 \%$ yield
Scheme 3 Doubly decarboxylative Giese reaction - reaction involving coumarin-3-carboxylic acids 2 .

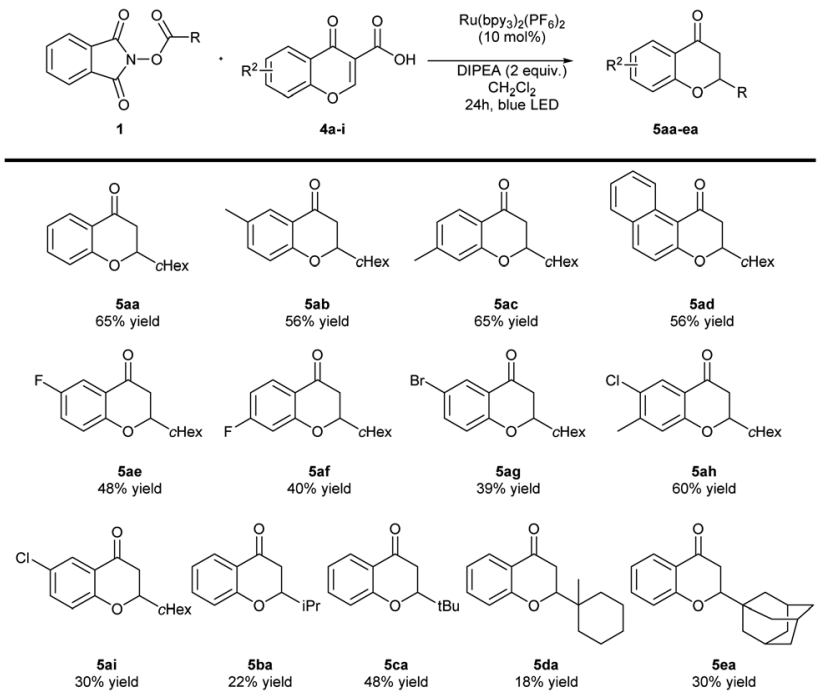

Scheme 4 Doubly decarboxylative Giese reaction - reaction involving chromone-3-carboxylic acids 4.

product 5ca was obtained with the highest yield in this part of the study. Unfortunately, the rest of examples showed lower responsiveness to participate in the reaction.

The postulated mechanism of both developed reactions is similar and begins with photocatalyst excitation (Scheme 5). Then the electron transfer from the amine to the photocatalyst takes place. Fluorescence quenching and cyclic voltammetry experiments confirmed the lack of quenching in the case of acids 2 a or $4 \mathbf{a}$ as well as $N$-(acyloxy)phthalimide 1a (for details see ESI $\dagger$ ). Subsequently, Ru(I) species acts as reductant of the $N$ (acyloxy)phthalimide 6. In turn, 7 undergoes decarboxylative

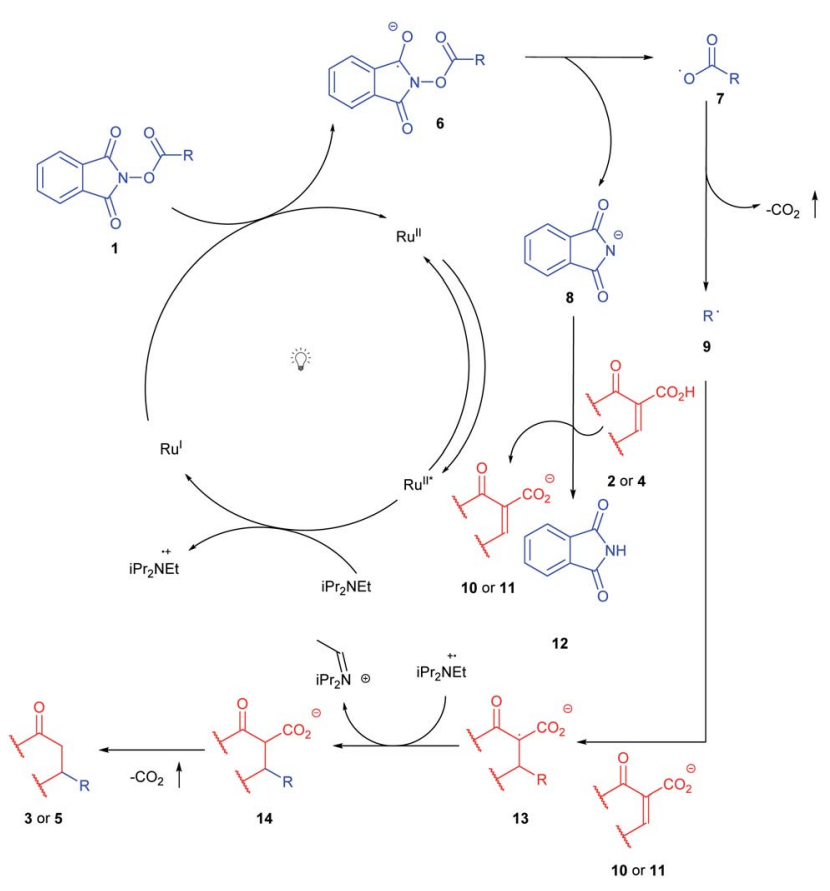

Scheme 5 Doubly decarboxylative Giese reaction - mechanism. 
degradation with the formation of an alkyl radical 9. The newly formed radical $\mathbf{9}$ undergoes Giese reaction with the acceptor $\mathbf{1 0}$ or 11. Transfer of the hydrogen atom from the radical cation originating from amine and subsequent decarboxylative protonation terminates the reaction affording $\mathbf{3}$ or $\mathbf{5}$ as target products. ${ }^{10 a}$

\section{Conclusions}

In conclusion, we have developed a doubly decarboxylative photocatalytic Giese reaction. It exemplifies the unique application of a free carboxylic-acid-activated olefins in a radical transformation. The reaction was applicable to a carboxylic acids as various coumarin-3-carboxylic acids $\mathbf{2 a - i}$ and chromone-3-carboxylic acids $\mathbf{4 a - i}$ served as effective Giese acceptors. The reaction can be described as doubly decarboxylation process with the first decarboxylation initiating the cycle and the second completing the process. Target, biologically relevant 4-substituted-chroman-2-ones 3aa-ea and 2substituted-chroman-4-ones 5aa-ea were obtained in good to high yields under mild reaction conditions.

\section{Conflicts of interest}

There are no conflicts to declare.

\section{Acknowledgements}

This work was financially supported by Ministry of Science and Higher Education Poland within the Diamond grant programme realized in the period 2018-2022, project number: 0016/DIA/2018/47. Thanks are expressed to Angelika Artelska and Adam Sikora (Faculty of Chemistry, Lodz University of Technology) for performing fluorescence quenching. This contribution has been completed while the second author (EK) was the Doctoral Candidate in the Interdisciplinary Doctoral School of Lodz University of Technology, Poland.

\section{References}

1 (a) D. P. Kamat, S. G. Tilve, V. P. Kamat and J. K. Kirtany, Syntheses and Biological Activities of Chroman-2-ones, Org. Prep. Proc. Int., 2015, 47, 1; (b) T. Okamoto, T. Kobayashi and S. Yoshida, Chemical aspects of coumarin compounds for the prevention of hepatocellular carcinomas, Curr. Med. Chem., 2005, 5, 47; (c) F. Asai, M. Iinuma, T. Tanaka and M. Mizuno, Complex flavonoids in farinose exudate from Pityrogramma calomelanos, Phytochemistry, 1991, 30, 3091.

2 (a) Ø. M. Andersen and K. R. Markham, Flavonoids: Chemistry, Biochemistry and Applications. CRC, Taylor \& Francis, Boca Raton, FL, 2006; (b) S. Emami and Z. Ghanbarimasir, Recent advances of chroman-4-one derivatives: synthetic approaches and bioactivities, Eur. J. Med. Chem., 2015, 93, 539; (c) K.-S. Masters and S. Bräse, Xanthones from fungi, lichens, and bacteria: the natural products and their synthesis, Chem. Rev., 2012, 112, 3717; (d) A. D. Patil, A. J. Freyer, D. S. Eggleston,
R. C. Haltiwanger, M. F. Bean, P. B. Taylor, M. J. Caranfa, A. L. Breen, H. R. Bartus and R. K. Johnson, The inophyllums, novel inhibitors of HIV-1 reverse transcriptase isolated from the Malaysian tree, Calophyllum inophyllum Linn, J. Med. Chem., 1993, 36, 4131; (e) D. L. Galinis, R. W. Fuller, T. C. McKee, J. H. II Cardellina, R. J. Gulakowski, J. B. McMahon and M. R. Boyd, Structure-Activity Modifications of the HIV-1 Inhibitors (+)-Calanolide A and (-)-Calanolide B1, J. Med. Chem., 1996, 39, 4507.

3 G. C. L. S. Ee, H. Mah, S. S. Teh, M. Rahmani, R. Go and Y. H. Taufiq-Yap, Soulamarin, a new coumarin from Stem Bark of Calophyllum soulattri, Molecules, 2011, 16, 9721.

4 Y. Shen, L. Wang, A. Khalil and Y. Kuo, Chromanones and dihydrocoumarins from Calophyllum blancoi, Chem. Pharm. Bull., 2004, 52, 402.

5 D. Genovese, C. Conti, P. Tomae, N. Desideri, M. Setin, S. Catone and L. Flore, Effect of chloro-, cyano-, and amidino-substituted flavanoids on enterovirus infection in vitro, Antiviral Res., 1995, 27, 123.

6 A. Suksamrarn, A. Chotipong, T. Suavansri, S. Boongird, P. Tirnsuksai, S. Vimuttipong and A. Chuaynugu, Antimycobacterial Activity and Cytotoxicity of Flavonoids from the Flowers of Chromolaena odorata., Arch. Pharmacal Res., 2004, 27, 507.

7 (a) B. Giese, J. A. Gonzalez-Gomez and T. Witzel, The Scope of Radical CC-Coupling by the "Tin Method", Angew. Chem., Int. Ed., 1984, 23, 69; (b) L. Chu, C. Ohta, Z. Zuo and D. W. C. MacMillan, Carboxylic Acids as A Traceless Activation Group for Conjugate Additions: A Three-Step Synthesis of ( \pm )-Pregabalin, J. Am. Chem. Soc., 2014, 136, 10886; (c) J. Schwarz and B. König, Metal-Free, VisibleLight-Mediated, Decarboxylative Alkylation of BiomassDerived Compounds, Green Chem., 2016, 18, 4743; (d) N. P. Ramirez and J. C. Gonzalez-Gomez, Decarboxylative Giese-Type Reaction of Carboxylic Acids Promoted by Visible Light: A Sustainable and Photoredox-Neutral Protocol, Eur. J. Org. Chem., 2017, 2154; (e) J. RostollBerenguer, G. Blay, J. R. Pedro and C. Vila, Photocatalytic Giese Addition of 1,4-Dihydroquinoxalin-2-ones to Electron-Poor Alkenes Using Visible Light, Org. Lett., 2020, 22, 8012; $(f)$ F. El-Hage, C. Schöll and J. Pospech, PhotoMediated Decarboxylative Giese-Type Reaction Using Organic Pyrimidopteridine Photoredox Catalysts, J. Org. Chem., 2020, 85, 13853.

8 (a) R. Brimioulle, D. Lenhart, M. M. Maturi and T. Bach, Enantioselective Catalysis of Photochemical Reactions, Angew. Chem., Int. Ed., 2015, 54, 3872; (b) L. Buzzetti, G. E. M. Crisenza and P. Melchiorre, Mechanistic Studies in Photocatalysis, Angew. Chem., Int. Ed., 2019, 58, 3730; (c) A. Vega-Peñaloza, J. Mateos, X. Companyó, M. EscuderoCaso and L. Dell'Amico, A Rational Aproach to OrganoPhotocatalysis Novel Designs and Structure-Property Relationships, Angew. Chem., Int. Ed., 2021, 60, 1082; (d) N. A. Romero and D. A. Nicewicz, Organic Photoredox Catalysis, Chem. Rev., 2016, 17, 10075; (e) M.-C. Fu, R. Shang, B. Zhao, B. Wang and Y. Fu, Photocatalytic 
decarboxylative alkylations mediated by triphenylphosphine and sodium iodide, Science, 2019, 363, 1429.

9 (a) S. K. Parida, T. Mandal, S. Das, S. K. Hota, S. De Sarkar and S. Murarka, Single Electron Transfer-Induced Redox Processes Involving $N$-(Acyloxy)phthalimides, ACS Catal., 2021, 11, 1640; (b) S. Shibutani, K. Nagao and H. Ohmiya, Organophotoredox-Catalyzed Three-Component Coupling of Heteroatom Nucleophiles, Alkenes, and Aliphatic Redox Active Esters, Org. Lett., 2021, 23, 1798; (c) Y. Dong, P. Ji, Y. Zhang, Ch. Wang, X. Meng and W. Wang, Organophotoredox-Catalyzed Formation of Alkyl-Aryl And -Alkyl C-S/Se Bonds from Coupling of Redox-Active Esters with Thio/Selenosulfonates, Org. Lett., 2020, 22, 9562; (d) T. Saget and B. König, Photocatalytic Synthesis of Polycyclic Indolones, Chem. Eur. J., 2020, 26, 7004; (e) C. Zheng, G.-Z. Wang and R. Shang, Catalyst-free Decarboxylation and Decarboxylative Giese Additions of Alkyl Carboxylates through Photoactivation of Electron Donor-Acceptor Complex, Adv. Synth. Catal., 2019, 361, 4500; (f) K. Okada, K. Okamoto, N. Morita, K. Okubo and M. Oda, Photosensitized decarboxylative Michael addition through $N$-(acyloxy)phthalimides via an electron-transfer mechanism, J. Am. Chem. Soc., 1991, 113, 9401; $(g)$ G.-Z. Wang, M.-C. Fu, B. Zhao and R. Shang, Photocatalytic decarboxylative alkylations of $\mathrm{C}\left(\mathrm{sp}^{3}\right)-\mathrm{H}$ and $\mathrm{C}\left(\mathrm{sp}^{2}\right)-\mathrm{H}$ bonds enabled by ammonium iodide in amide solvent, $\mathrm{Sci}$. China Chem., 2021, 64, 439; $(h)$ M. Tian, Y. Wang, X. Bu, Y. Wang and X. Yang, An ultrastable olefin-linked covalent organic framework for photocatalytic decarboxylative alkylations under highly acidic conditions, Catal. Sci. Technol., 2021, 11, 4272.

10 (a) K. N. Tripathi, Md. Belal and R. P. Singh, Organo Photoinduced Decarboxylative Alkylation of Coumarins with N-(Acyloxy)phthalimide, J. Org. Chem., 2020, 85, 1193; (b) S. Das, S. Kumar Parida, T. Mandal, S. K. Hota, L. Roy, S. D. Sarkar and S. Murarka, An organophotoredoxcatalyzed redox-neutral cascade involving $\mathrm{N}$-(acyloxy) phthalimides and maleimides, Org. Chem. Front., 2021, 8, 2256.

11 (a) J.-J. Zhang, J.-C. Yang, L.-N. Guo and X.-H. Duan, VisibleLight-Mediated Dual Decarboxylative Coupling of RedoxActive Esters with $\alpha, \beta$-Unsaturated Carboxylic Acids,
Chem.-Eur. J., 2017, 23, 10259; (b) K. Xu, Z. Tan, H. Zhang, J. Liu, S. Zhang and Z. Wang, Photoredox catalysis enabled alkylation of alkenyl carboxylic acids with $N$-(acyloxy) phthalimide via dual decarboxylation, Chem. Commun., 2017, 53, 10719; for reviews on decarboxylative strategies, see: ; (c) Y. Pan and C.-H. Tan, Catalytic Decarboxylative Reactions: Biomimetic Approaches Inspired by Polyketide Biosynthesis, Synthesis, 2011, 2044; (d) Z.-L. Wang, Recent Advances in Catalytic Asymmetric Decarboxylative Addition Reactions, Adv. Synth. Catal., 2013, 355, 2745; (e) S. Nakamura, Catalytic enantioselective decarboxylative reactions using organocatalysts, Org. Biomol. Chem., 2014, 12, 394; $(f)$ J. Bojanowski and A. Albrecht, Carboxylic-Acid-Activated Olefins in Decarboxylative Reactions, Asian J. Org. Chem., 2019, 8, 746.

12 (a) L. Xu, Z. Shao, L. Wang and J. Xiao, Tandem sp3 C-H Functionalization/Decarboxylation of 2-Alkylazaarenes with Coumarin-3-carboxylic Acids, Org. Lett., 2014, 16, 796; (b) F. Han, S. Xun, L. Jia, Y. Zhang, L. Zou and L. Hu, Traceless-Activation Strategy for Rh-Catalyzed Csp2 - H Arylation of Coumarins, Org. Lett., 2019, 21, 5907.

13 (a) Z. Shao, L. Wang, L. Xu, H. Zhao and J. Xiao, Facile synthesis of azaarenes-2-substituted chromanone derivatives via tandem $\mathrm{sp} 3 \mathrm{C}-\mathrm{H}$ functionalization/ decarboxylation of azaarenes with 4-oxo-4H- chromene-3carboxylic acid, RSC Adv., 2014, 4, 53188; (b) A. G. Neo, J. Diaz, S. Marcaccinib and C. F. Marcos, Conjugate addition of isocyanides to chromone 3-carboxylic acid: an efficient one-pot synthesis of chroman-4-one 2carboxamides, Org. Biomol. Chem., 2012, 10, 3406.

14 (a) S. Nakamura, A. Toda, M. Sano, T. Hatanaka and Y. Funahashi, Organocatalytic Enantioselective Conjugate Addition of Malonic Acid Half Thioesters to Coumarin-3-carboxylic Acids Using $N$-Heteroarenesulfonyl Cinchona Alkaloid Amide, Adv. Synth. Catal., 2016, 358, 1029; (b) S. Peng, L. Wang, H. Guo, S. Sunb and J. Wang, Facile synthesis of 4-substituted 3,4-dihydrocoumarins via an organocatalytic double decarboxylation process, Org. Biomol. Chem., 2012, 10, 2537; (c) A. Albrecht, Utilization of Chromone-3-Carboxylic Acids as Acceptors in the Michael-Type Decarboxylative Addition, Eur. J. Org. Chem., 2018, 648. 